\title{
Production cross sections of strange and charmed baryons at Belle
}

\author{
Mizuki Sumihama*† \\ Gifu University \\ E-mail: sumihamaercnp.osaka-u.ac.jp
}

Production cross sections of strange and charmed baryons in the $e^{+} e^{-}$annihilation are measured using the data collected with the Belle detector at $\sqrt{s}=10.52 \mathrm{GeV}$. Hadron production rates lie on a simple function of their masses due to a linear potential. The study of production rates of various states will provide information on the quark structure of hadrons. If a production rate of a hadron is deviated from the normal mass dependence, it may be "exotic" in terms of a quark structure. Information on a di-quark picture will be given from production rates of charmed baryons and strange baryons. The preliminary results are presented.

XV International Conference on Hadron Spectroscopy-Hadron 2013

4-8 November 2013

Nara, Japan

*Speaker.

${ }^{\dagger}$ On behalf of Belle Collaboration 


\section{Introduction}

Production rates for light flavored hadrons were studied in the $e^{+} e^{-}$annihilation process at $\sqrt{s} \sim 10 \mathrm{GeV}$ at ARGUS [1, 2], and CLEO [3] shown in Fig. 1. The production rate for light

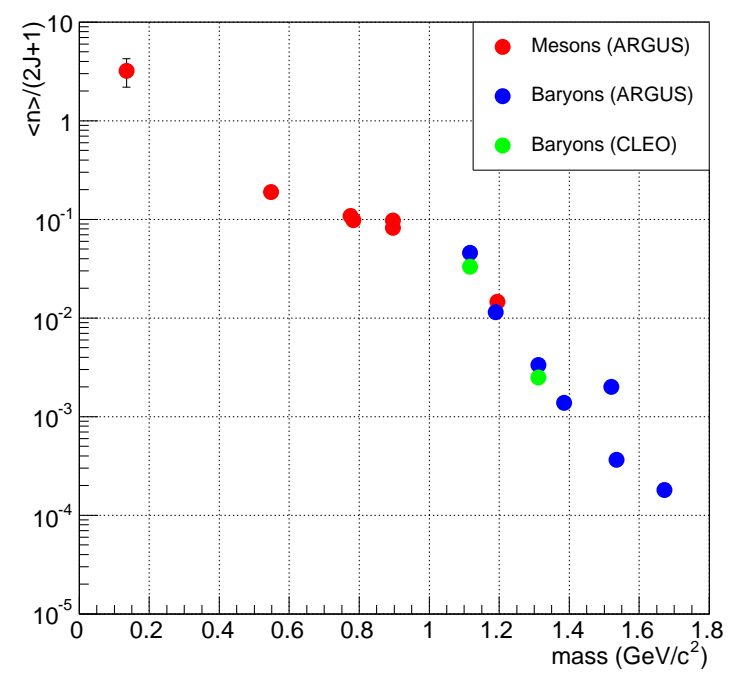

Figure 1: Production rate of light mesons and baryons from ARGUS and CLEO at $\sqrt{s} \sim 10$

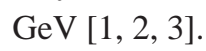

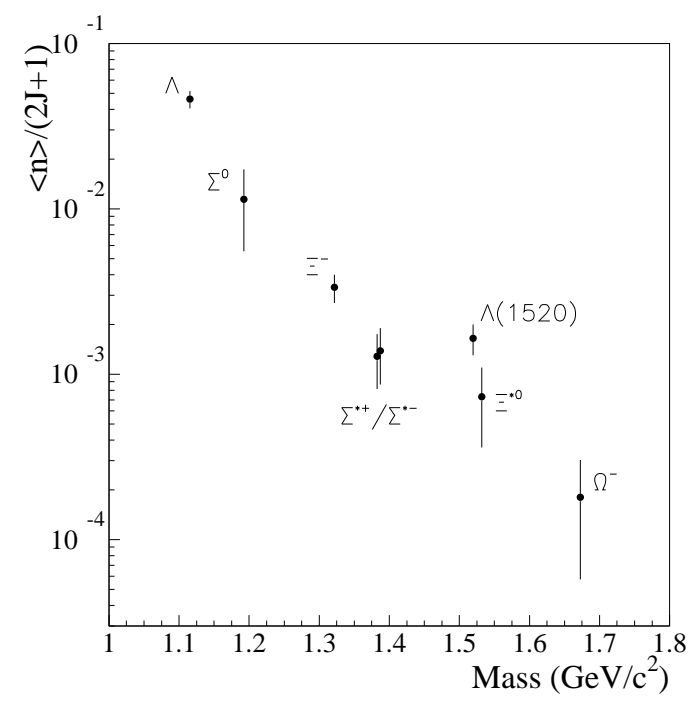

Figure 2: Production rate of strange baryons from ARGUS at $\sqrt{s} \sim 10 \mathrm{GeV}$ [近, 凤.

hadrons is described as

$$
\frac{<n>}{2 J+1} \propto e^{-\alpha m}
$$

where $\langle n\rangle, J, m$ and $\alpha$ are the averaged number, the total spin, and the mass of hadrons, and,

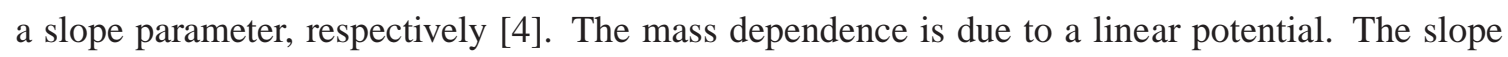
parameter, $\alpha$, is different between mesons and baryons as shown in Fig. 1. It is expected to be due to the constituent quark counting, $q \bar{q}$ for mesons, and $q q q$ for baryons 惯. The production rate for "exotic" baryons, for example $\Lambda(1405)$, and $\Theta^{+}$pentaquark, may not lie on a slope of the normal baryon if the quark structure is not three [阿]. Exotic baryons may be shown by the systematic studies of production rates. Figure $\mathrm{Z}$ shows the mass dependence of production rates for strange baryons obtained at ARGUS [1],2]. The production rate of $\Lambda(1520)$ deviates from other baryons. The deviation may indicate an unusual quark structure of $\Lambda(1520)$. The spin-parity and the orbital angular momentum of $\Lambda(1520)$ is $3 / 2^{-}$, and 1 , respectively. The production rate of $\Lambda(1405)$ with the spin-parity of $1 / 2^{-}$, and $L=1$ will provide information on the deviation of $\Lambda(1520)$. There is an analogical pair of charmed baryons. The spin-parity of $\Lambda_{c}(2595)^{+}$and $\Lambda_{c}(2625)^{+}$is $1 / 2^{-}$ and $3 / 2^{-}$, respectively. Both particles have the same orbital angular momentum, $L=1$ [6]. The precise systematic study of strange and charmed baryons is interesting to search for exotic baryons and understand their quark structure.

To know the structure of hadrons, we need to understand how quarks interact each other in hadrons. In case of strange baryons, SU(3) symmetry works well. The 3 quarks or 3 pairs of 
quarks are uniformly interacted each other in baryons. In case of charmed baryons, the situation is different because of the heavy mass of a charm quark. A di-quark correlation in a charmed baryon is strong due to a weak color magnetic interaction with a heavy quark of a charm. The quark structure of charmed baryons is described by a di-quark picture which is developed by a pair of light quarks and a single heavy quark. A pair of light quarks with $S=0(S=1)$ is called a good (bad) di-quark [7]. The production rate of a good di-quark is expected to be higher than that of a bad di-quark because the interaction between two quarks in a good di-quark is stronger than that in a bad-quark due to the spin-spin interaction. We expect higher production rate for $\Lambda_{c}^{+}$including a good di-quark than that for $\Sigma_{c}$ including a bad di-quark. It is interesting and important to see the tendency of production rates for strange baryons and charmed baryons. We present the preliminary result of the production rates for $\left.\Lambda, \Lambda(1520), \Sigma^{0}, \Sigma^{*+}(1385), \Xi^{-}, \Omega^{-}, \Omega_{c}, \Lambda_{c}^{+}, \Sigma_{c}^{(} 2455\right) 0, \Lambda_{c}(2625)^{+}$, and $\Sigma_{c}(2520)^{0}$ particles.

\section{Analysis}

The production rate is obtained using the data corrected at the total energy of $\sqrt{s}=10.52 \mathrm{GeV}$ where is the off region of the $\Upsilon(4 S)$ resonance. The integrated luminosity is $79.366 \mathrm{fb}^{-1}$. The data collected at the $\Upsilon(4 S)$ resonance is used to check the analysis procedure and see the contribution from the B-decay.

A good vertex reconstruction is achieved by installing the silicon vertex detector in $2 \mathrm{~cm}$ from the interaction point, and using light materials for tracking devices. The resolution of a reconstructed vertex for $B \rightarrow J / \psi K_{S}$ is about $80 \mu \mathrm{m}$. The detector configuration is good for the analysis of hyperons which are long-life particles. The decay points of $\Xi$ and $\Omega$ particles, and their daughter particle, $\Lambda$, are not always the interaction point. The good vertex reconstruction is required to see these long-life particles.

Table 1: Baryons and decay processes in the current study. For anti-baryon, the charge conjugate condition is used.

\begin{tabular}{|c|c|}
\hline Baryon & Decay Process \\
\hline \hline$\Lambda$ & $p \pi^{-}$ \\
\hline$\Lambda(1520)$ & $p K^{-}$ \\
\hline$\Sigma^{0}$ & $\Lambda \gamma \rightarrow p \pi^{-} \gamma$ \\
\hline$\Sigma(1385)^{+}$ & $\Lambda \pi^{+} \rightarrow p \pi^{-} \pi^{+}$ \\
\hline$\Xi^{-}$ & $\Lambda \pi^{-} \rightarrow p \pi^{-} \pi^{-}$ \\
\hline$\Omega^{-}$ & $\Lambda K^{-} \rightarrow p \pi^{-} K^{-}$ \\
\hline$\Lambda_{c}^{+}$ & $p K^{-} \pi^{+}$ \\
\hline$\Lambda_{c}^{+}(2625)$ & $\Lambda_{c}^{+} \pi^{+} \pi^{-} \rightarrow p K^{-} \pi^{+} \pi^{+} \pi^{-}$ \\
\hline$\Sigma_{c}^{0}(2455)$ & $\Lambda_{c}^{+} \pi^{-} \rightarrow p K^{-} \pi^{+} \pi^{-}$ \\
\hline$\Sigma_{c}^{0}(2520)$ & $\Lambda_{c}^{+} \pi^{-} \rightarrow p K^{-} \pi^{+} \pi^{-}$ \\
\hline$\Omega_{c}^{0}$ & $\Omega^{-} \pi^{+} \rightarrow \Lambda\left(p \pi^{-}\right) K^{-} \pi^{+}$ \\
\hline
\end{tabular}


The baryons and decay processes studied in this work are listed in Table 1. The charge conjugate condition is used for an anti-baryon. The differential cross sections for a baryon and an anti-baryon using the data collected at the $\Upsilon(4 \mathrm{~S})$ resonance and the data at the off resonance region are obtained as a function of a scaled momentum $x_{p}=p_{c . m .} / \sqrt{\frac{s}{4}-M^{2}}$, where $p_{c . m}$. is the $c . m$. momentum of hadrons with a mass $M$, and $s$ is a $c . m$. energy. $x_{p}$ is a ratio of the momentum to the maximum possible momentum of hadrons.

The signal yields of a baryon and an anti-baryon are obtained in each decay process by correcting for the detector acceptance which is estimated using a Monte Carlo simulation. The total cross sections are calculated by dividing the signal yields by the integrated luminosity, and the branching ratio of the analyzed decay process. The measured cross section includes events by decay processes of higher states (feed-down process). The direct cross section by the primary production is calculated by subtracting these feed-down processes from the measured cross sections. The considered feed-down processes for each baryon are summarized in Table 2 . The cross sections of feed-down processes are measured in this work. The total cross sections for $\Xi^{-}$and $\Sigma^{*+}$ are obtained, but not for $\Xi^{0}$ and $\Sigma^{*-, 0}$. The isospin symmetry was assumed for production cross sections of $\Sigma^{*}$, and $\Xi$.

Table 2: Feed-down processes taken into account to calculate the direct cross sections.

\begin{tabular}{|c|c|}
\hline Baryon & Feed-down \\
\hline $\bar{\Lambda}$ & $\begin{array}{c}\Sigma^{0} \\
\Sigma(1385)^{-, 0,+} \\
\Xi^{-}, \Xi^{0} \\
\Lambda(1520) \\
\Lambda_{c}^{+}\end{array}$ \\
\hline$\Sigma^{0}$ & $\begin{array}{c}\Sigma(1385)^{-,+} \\
\Lambda(1520) \\
\Lambda_{c}^{+}\end{array}$ \\
\hline$\Sigma(1385)^{+}$ & $\Lambda(1520)$ \\
\hline$\Lambda(1520)$ & $\Lambda_{c}^{+}$ \\
\hline$\Xi^{-}$ & - \\
\hline$\Omega^{-}$ & $\Omega_{c}$ \\
\hline$\Lambda_{c}^{+}$ & $\begin{array}{c}\Sigma_{c}(2455)^{0,+,++} \\
\Sigma_{c}(2520)^{0,+,++} \\
\Lambda_{c}(2625)^{+}\end{array}$ \\
\hline$\Lambda_{c}^{+}(2625)$ & - \\
\hline$\Sigma_{c}^{0}(2455)$ & - \\
\hline$\Sigma_{c}^{0}(2520)$ & - \\
\hline$\Omega_{c}^{0}$ & - \\
\hline
\end{tabular}

Figure 3 shows the preliminary result of production rates for strange and charmed baryons as a function of a baryon mass. The closed circles are the results from this work. The opened circle is the data from the other work of Belle [8]. The obtained cross sections $(\sigma)$ are normalized by the 


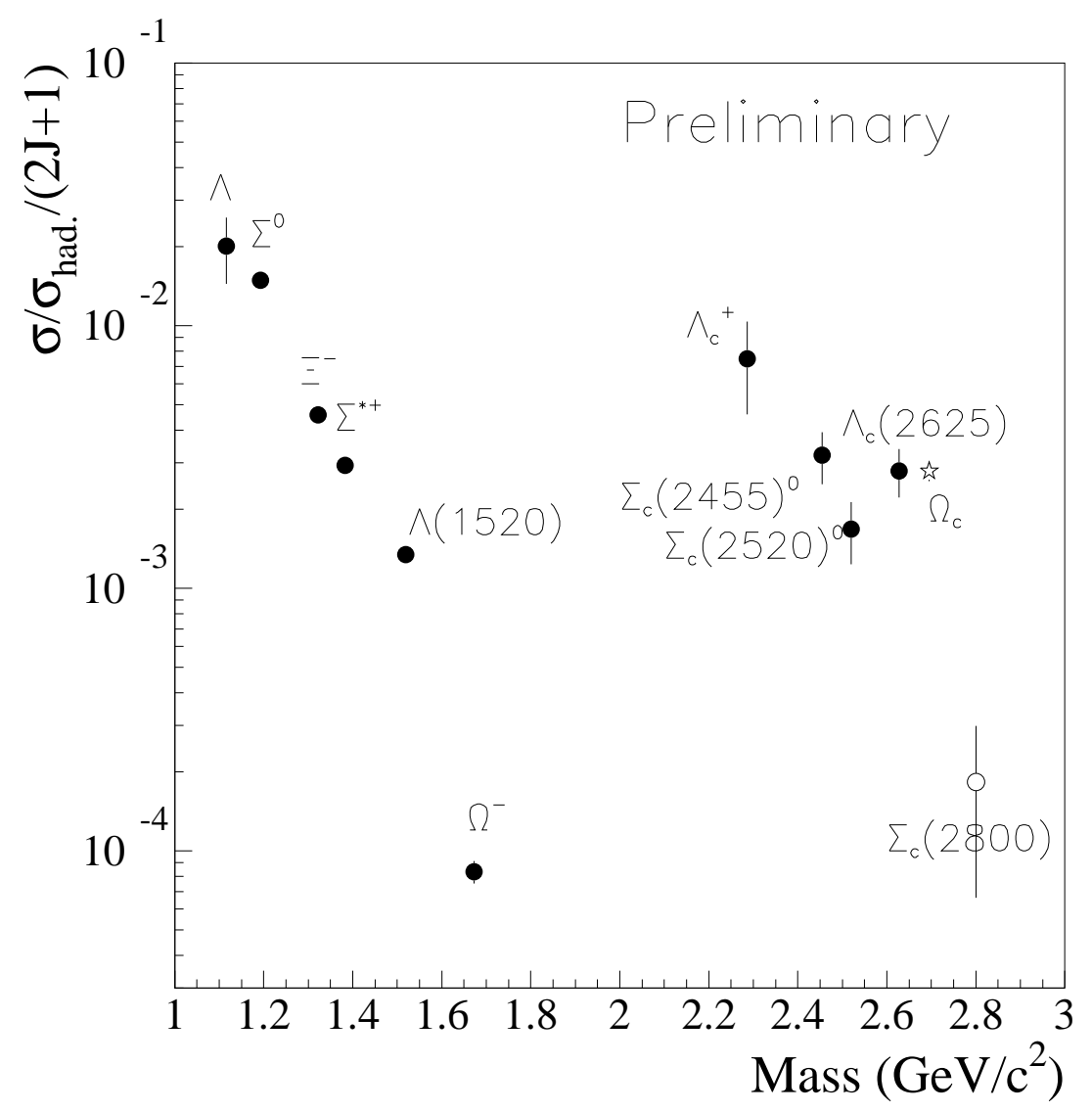

Figure 3: Preliminary result of production rates as a function of a mass of baryons.

total hadronic cross section, and the number of spin states. The spin is from Particle Data group[6]. The total hadronic cross section, $\sigma_{\text {had }}$, was obtained by the CLEO collaboration at $\sqrt{s}=10.52$ $\mathrm{GeV}$ which is the same energy of this work. The $\sigma_{\text {had }}$ corrected for the initial state radiation and electron-positron vertex loops is $2.794 \mathrm{nb}$ [6, 9]. The production rate, $\langle\mathrm{n}>$, is obtained as $\sigma /$ $\sigma_{\text {had }}$. The statistics is good and the errors are mainly by the correction for feed-down processes. The production rate of $\Omega_{c}$ is obtained using the branching fraction of $\Omega_{c} \rightarrow \Omega \pi,(0.25 \pm 0.12) \%$. This number has been obtained by following PDG's estimation of the branching fraction of the $\Lambda_{c}^{+} \rightarrow p K^{-} \pi^{+}$decay [6].

\section{Discussion and outlook}

The mass dependence of strange baryons is different from that of charmed baryons because of a difference of a production rate for a primary $c \bar{c}$ pair and $s \bar{s}$ pair in the $e^{+} e^{-}$annihilation. The discrepancy between the result of this work and ARGUS for $\Lambda$, and $\Sigma^{*}$ is large. It may be due to a difference of the treatment for feed-down processes. The deviation of $\Lambda(1520)$ is not clear like the ARGUS data. The production rate of $\Omega$ is lower than other strange baryons which is expected to be due to the strange suppression factor, or no good di-quark in $\Omega$ particle [ 4 . A series of charmed baryons shows interesting features. They do not line on the same line. It can be explained that 
the production rates of two $\Lambda_{c}^{+}$'s is higher than $\Sigma_{c}$ particles due to the di-quark picture of charmed baryons. The production rate of a good di-quark $\left(\Lambda_{c}^{+}\right)$is expected to be higher than the production rate of a bad di-quark $\left(\Sigma_{c}\right)$ because of a stronger interaction between quarks in a good di-quark than a bad di-quark. From the other point of view, only $\Lambda_{c}(2625)^{+}$seems to be deviated from other $\Lambda_{c}^{+}$, and $\Sigma_{c}$ baryons. The production rate of $\Lambda_{c}(2595)^{+}$may provide more information on the mass dependence. The mass difference between $\Lambda_{c}(2595)^{+}$and $\Lambda_{c}(2625)^{+}$is only $33 \mathrm{MeV}$. The spin-parity of $\Lambda_{c}(2595)^{+}$and $\Lambda_{c}(2625)^{+}$is $1 / 2^{-}$and $3 / 2^{-}$. The orbital angular momentum of both particles is 1 . The production rate for $\Omega_{c}$ is deviated from other charmed baryons. The systematic study of charmed baryons will evaluate a predicted branching ratio.

We proceed the data analysis to obtain the final result of the mass dependence of production rates including measurement of cross sections of baryons other than presented in this report.

\section{Summary}

Production cross sections of strange and charmed baryons in the $e^{+} e^{-}$annihilation are measured at $\sqrt{s}=10.52 \mathrm{GeV}$. The precise measurement of production rates is possible to thank for the good statistics and the good performance of the Belle detector. The mass dependence of production rates is good mean to search for exotic hadrons because the mass dependence is due to the quark counting.

The comparison between strange and charmed baryons is interesting and important to see the di-quark picture. A di-quark correlation in a charmed baryon is stronger than that of strange baryons. The production rate for $\Lambda_{c}^{+}$including a good di-quark is expected to be higher than that for $\Sigma_{c}$ including a bad di-quark. This feature may not seen in strange baryons because of its weak di-quark correlation. The wide variation of the mass dependence is seen in charmed baryons but not in strange baryons. The further study of production rates of many bary ons with various spin-parity is proceeded to see their quark structures.

\section{References}

[1] H. Albrecht et al., Z. Phys. C 39 (1988) 177.

[2] H. Albrecht et al., Phys. Lett. B 215 (1988) 429.

[3] S. Behrends et al., Phys. Rev. D 31 (1985) 2161.

[4] Y.J. Pei, Z. Phys. C 72 (1996) 39.

[5] V. Halyo, TALK-04/095 Pentaquark Studies in BaBar.

[6] Particle Data Group, Phys. Rev. D 86 (2012) 010001.

[7] R. L. Jaffe, Phys. Rept. 409 (2005) 1-45,

[8] R. Mizuk et. al. (Belle Collaboration), Phys. Rev. Lett. 94 (2005) 182002.

[9] R. Ammar et al., Phys. Rev. D 57 (1998) 1350. 